\title{
The Application of Cloud Computing in the Enterprise Credit Rating
} System

\author{
Xuyan $\mathrm{CHUN}^{1, a}$, Xieshu FANG ${ }^{2, b}$ \\ ${ }^{1}$ Department of Information and Art, Shandong Kaiwen College of Science and Technology, Jinan, \\ 250200, China \\ ${ }^{2}$ Department of Information and Art, Shandong Kaiwen College of Science and Technology, Jinan, \\ 250200, China \\ ${ }^{a}$ E-mail: xu_yanchun@126.com, ${ }^{b}$ E-mail: xieshufang@sohu.com
}

Keywords: Credit Rating; Cloud Computing Technology; Resource Virtualization

\begin{abstract}
With the development of Internet banking and big data, the traditional credit rating in the sample data collection, model development, systems use and other aspects is affected. It mainly appears in the full quantization of data samples and enterprise behavior data which is unstructured, virtualization requirements of desktop etc.. In recent years, more and more mature cloud computing technology can meet the distributed call resources, large data mining, large data processing capabilities and resource virtualization capabilities.
\end{abstract}

\section{Introduction}

Traditional enterprise credit rating has a lot of problems: (1) Credit rating model is established through small sample data and it's difficult to collect full amount of data of enterprise management and behavior. So there is a very big one-sidedness. (2) Influenced by data collection and data processing technology, the information asymmetry problem has not been solved; (3) Measurement credit rating main scale of small sample data assumed that customers' defaults and losses obeyed normal distribution. Actually normal distribution of small sample data does not exist and only full amount of data and behavioral data analysis are close to normal distribution and the deviation of the measurement result will be reduced.

With the development of Internet technology and computer level, considerable progress has been made in large data collection and processing. The emergence of cloud computing technology can provide great technical support for credit rating.

\section{Cloud Computing and Credit Rating}

\subsection{The Solution to the Problem of Credit Rating}

Traditional credit rating based on the Internal data of an institution or a bank to establish a model, therefore, the application is also within the region or the bank. The problems are mainly concentrated on the aspects of model construction, information early warning and system operation. These problems can be solved by learning from big data and adopting cloud computing technology.

(1) Model Construction: In order to solve the problem of traditional credit rating model based on the small sample data affecting model accuracy, cloud computing architecture technology can be adopted to collect a large number of enterprise data and behavioral data, while allowing full amount of samples to obey normal distribution, accordingly prediction of credit rating can be reflected.

(2) In the traditional credit rating, a small number and breadth of collected information resulted in information asymmetry, and the new credit risk appears after solving a credit risk. To solve this problem, we can use cloud computing technology, integration of government, industry and commerce, taxation, water, electricity and other data, and even we can integrate the transaction information of the Internet and make a early warning of an enterprise credit risk.

(3) System Operation: Adoption of cloud virtual desktop can solve the problem of credit rating system, for example, the user's poor experience in the operation which is difficult to integrate data, 
and high cost of system's repeated construction, also can meet a number of banks, nonbank financial institutions and the public to operate or query.

\subsection{Integration of Cloud Computing Combined With Big Data and Credit Rating}

Using cloud computing combined with large data in the credit rating, Internet banking companies go in the forefront. Through the accumulation of a large number of customer information and behavior information, credit rating has made a lot of breakthroughs. Cloud computing technology combined with big data can promote the development of credit rating, with the possibility of integration and feasibility.

Two trends of Cloud computing technology development: one is the terminal extension, and the other is the background of cross-border integration. Through various types of cloud terminals, wireless access and other technologies, the terminal broke through the boundaries of equipment and formed cloud virtual desktop.

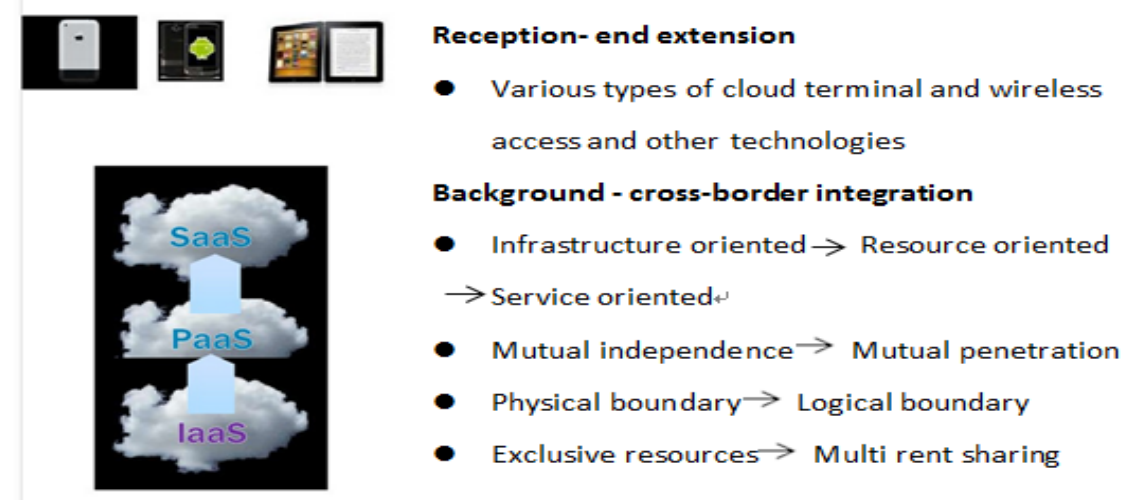

Fig.1. Front Background Extension

Under these two development trends, many financial institutions have to explore the possibility of the establishment of financial cloud. Cloud computing in the integration of large data and credit rating will appear in the form of financial cloud. Financial cloud will provide computer resources and operational services for banks and other financial institutions.

On the basis of mass enterprise data, the numerous mathematical statistical analysis model, assisted banks and other financial institutions for credit decision-making includes three levels: cloud-based platform, cloud decision-making platform and a top level business layer. Cloud platform, which is mainly based on cloud equipment and cloud operating systems, establish a cloud computing environment and create new services for the construction of the cloud. Cloud platform of decision-making is the core module, carrying on the comprehensive management of all kinds of resources, services, news, simulation test and so on, mainly through the calculation of the engine. Business layer, mainly built on top of the cloud strategy platform, includes credit marketing, credit rating, credit risk management, early warning collection, anti fraud and other business applications.

\subsection{The Significance of Cloud Computing to Credit Rating}

The significance of Cloud computing in the field of credit rating can be described by cloud computing services provided. General services for the financial industry are mainly reflected in: cloud resources services, cloud platform operation management services, cloud migration services, cloud security services, cloud emergency services and so on.

Cloud resources services build the cloud resource management platform using cloud computing technology. It can collect and integrate the external data of financial institutions, realize the big data management, and accumulate enough data, which is significant for transfer credit rating. In cloud operations management services, cloud computing platform can be built in accordance with the financial industry norms and processes to ensure compliance, legal and operational management of the smooth. Cloud security services establish the system of information security management services through the security mechanism of cloud platform. At present, cloud computing technology is mature and gradually meet the requirements of financial institutions for cloud security. Cloud backup services, a solution in the field of data pure technology, is one of the main means to ensure that the data will not be lost and there is no interruption of service. 
Calculation of the specific meaning of enterprise credit rating from the cloud computing, can use "multi, fast, good and province" to summarize. The "multi" refers to the large data collection and integration of cloud computing and processing capacity, which can be a full data credit rating modeling and full data risk warning (through the credit process); The "Fast" refers to the fact that cloud computing technology can make the credit rating model at any time to validate and adjust, the enterprises to monitor the real-time behavior; through cloud desktop, ratings and rating result query operation, greatly improving the speed of information query. The "Good" refers to the adoption of cloud computing technology to build a credit rating platform, rating models, risk warning and others timely and accurately, and can be more effective in the prevention and control of credit risks. "Province" refers to the use of cloud computing technology in the construction of the credit rating platform and can be a number of financial institutions sharing cloud services, from the bottom stratus devices to cloud services, therefore, construction cycle is short, and investment cost is low, safe and reliable, which can greatly reduce the construction cost of the financial institutions.

\section{Application of Cloud Computing in Credit Rating}

\subsection{Feasibility and Necessity of Application}

With the rapid development of cloud computing, big data, on the one hand, cloud computing has been given a bright future. Internet giant and the major manufacturers have joined the "national sport", tens of billions of funds flowing into the field of cloud computing continuously and constantly, depicting the blueprint after the implementation of cloud computing technology; people began to gradually accept the idea of cloud computing, and consider the relationship between cloud computing and its IT application; government cloud computing become an important driving force, began to layout of the upstream and downstream industry chain and promote the cloud computing industry development by leaps and bounds; domestic and foreign media are in scrambling to describe the unique charm of cloud computing; optimistic data is even more exciting, for 2015 global cloud computing market value exceeded \$121.1 billion. People for cloud computing is in an optimistic imagination, and the entire industry is very large.

At present, banks and institutions have established a large number of credit rating system, involving a large number of network devices, servers and storage devices. Most of the equipment utilization is low, and some of the work efficiency is below $20 \%$. In this way, wasted a lot of costs, resulted in the repeated constructional application. If these devices are integrated into cloud computing platform, the efficiency of the use of the server will be greatly enhanced (40\% 60\%), to be dynamic, flexible, and can be recycled to provide services for credit rating.

\subsection{Application of Technical Scheme}

The overall planning or design of the cloud platform is divided into three centers: local data center, local backup center, and remote disaster recovery center. The credit rating of the cloud platform in accordance with the actual network structure can be divided into four sets of network: cloud platform in the intranet, cloud platform in the network, image network, and credit rating business network. The network planning area is the following:

(1) Cloud platform in the intranet: core exchanging district, cloud resource pool area, function test district, operation and maintenance management district, outreach safe access area, local backup area, different content areas, ownership units access area, and cloud computing center.

(2) Cloud platform in the network: core exchanging district, cloud resource pool area, function test district, operation and maintenance management district, outreach safe access area, Internet access local backup area, different content areas, and ownership units access area.

(3) Image network: core exchange district, cloud resource pool area, function test district, operation and maintenance management district, outreach safe access area, local backup area, different content areas, and ownership units access area.

(4) Credit rating business private network: core business network security access area, exchange area, and server resources. 


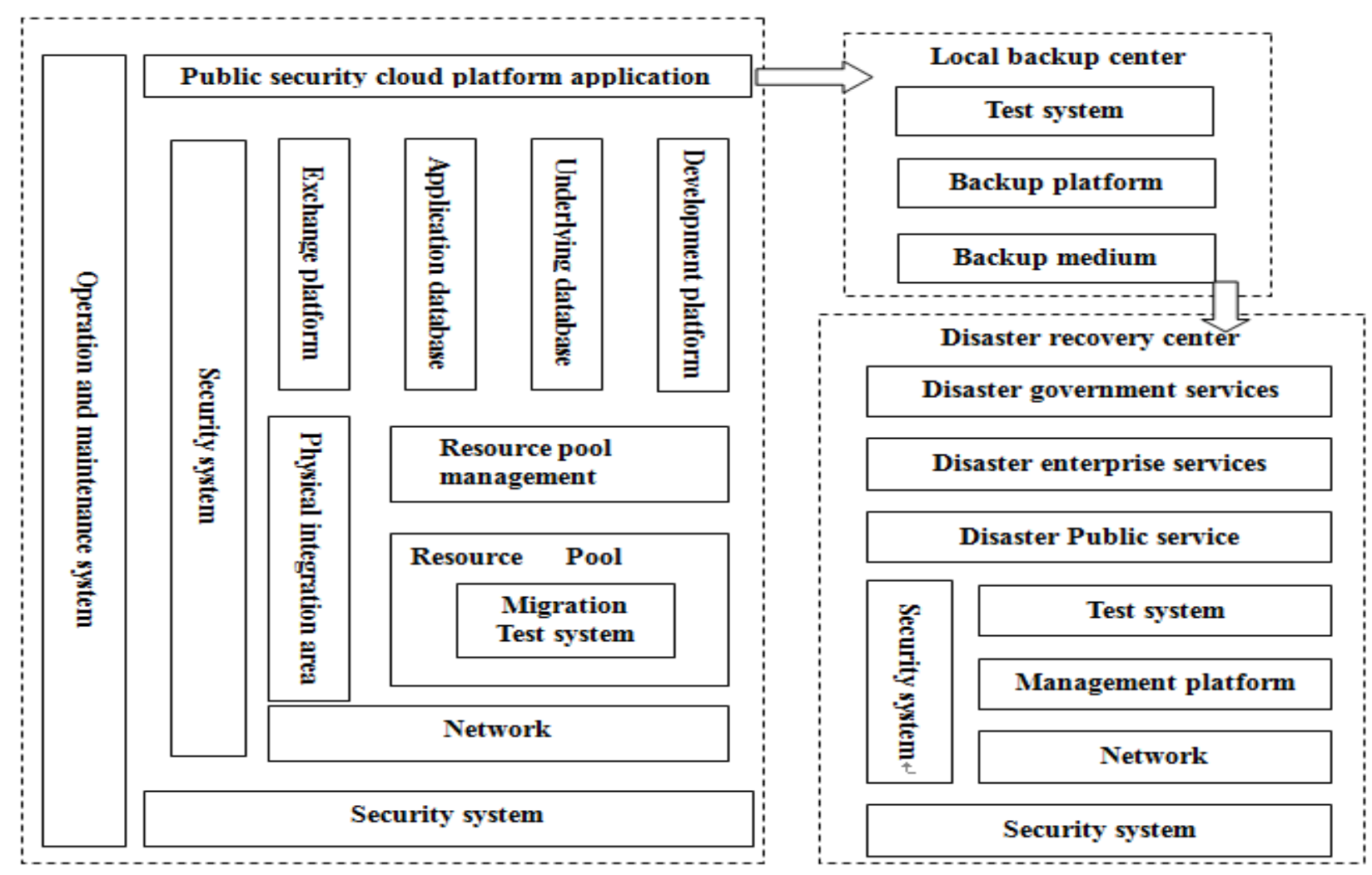

\subsection{Cloud Computing Security}

Fig. 2. Logical Design of Cloud Computing

The IaaS layer is mainly physical and environmental safety, including room physical circuit and host security, network security, virtualization security, storage security etc..

PaaS layer security is mainly for the security of data transmission and computing.

SaaS layer security includes the application security.

In addition to the layers of security, there are part of the public information security, including data security, encryption and secret key management, and dynamic password identity authentication system. Data security is particularly important. For the existence of structured data in Oracle, MSSQL, MySQL as the database, achieve data access security through the database security audit system; for the semi structured data and unstructured data through data encryption achieve data security.

\subsection{Using Virtualization Technology to Solve the Application of Credit Rating System}

For the development of credit rating model is mainly by cloud management platform, collect government, industry and commerce, taxation, electric power and other various aspects of data, form large data; and develop rating model using cloud computing technology of data processing and mining capacity.

After model development, directly in the big data environment, verify and pressure test, inspect and adjustment model. 


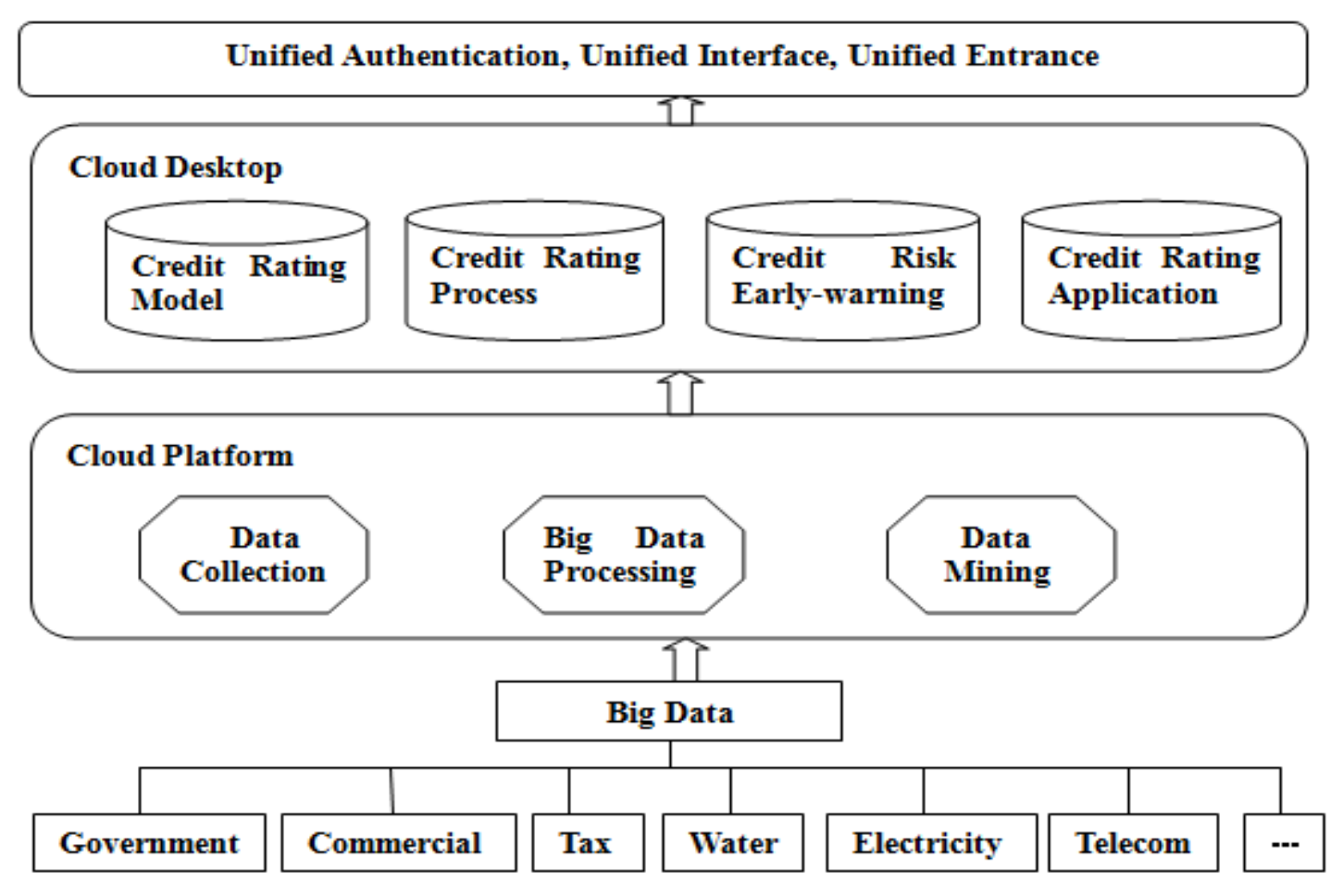

Fig.3. Cloud Computing Applications in Credit Rating

The credit rating of the cloud computing system includes: the Internet is the main part of the bridge of the server and the client. Cloud computing server group: the part of receiving user credit rating model configuration commands, in accordance with the requirements of the client, choose the corresponding template and template adjustment, and the new template is applied to the system for client use. Client: customer rating tool.

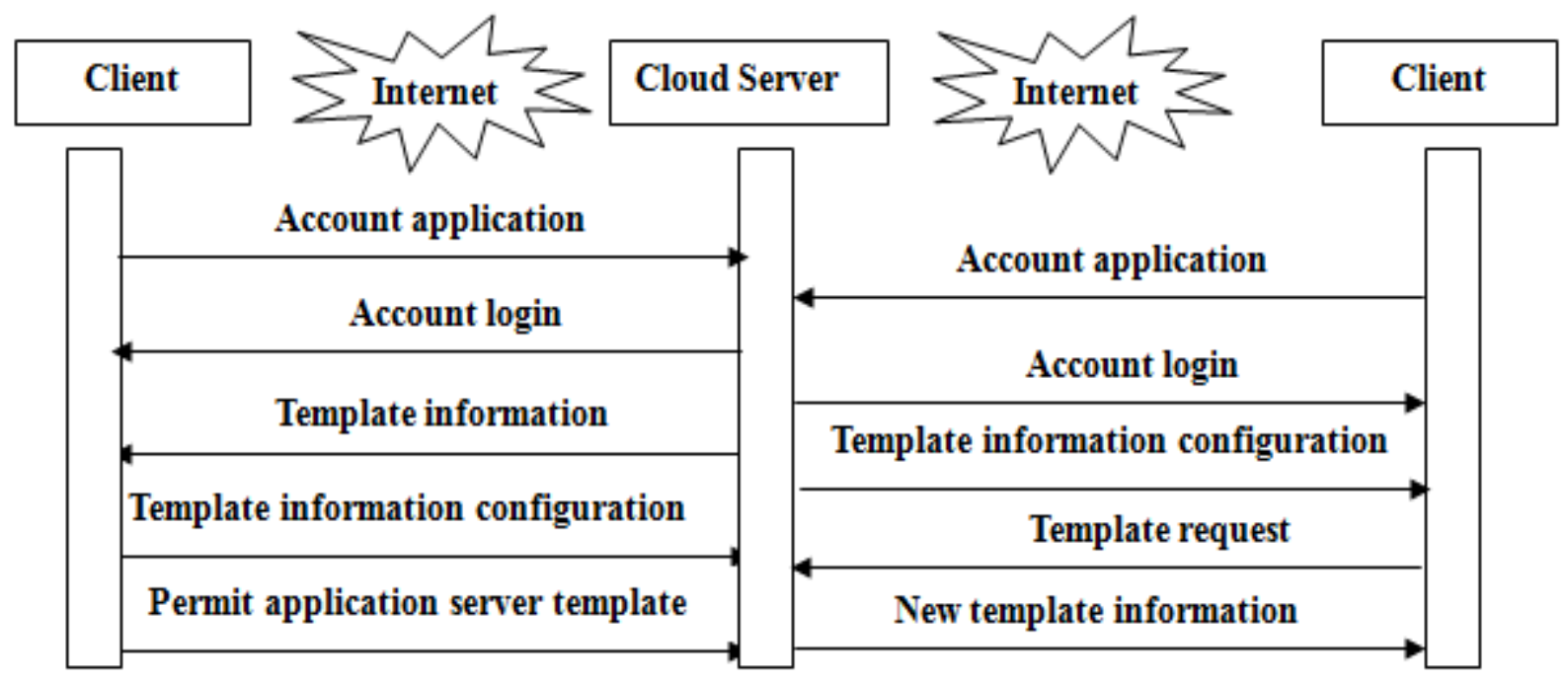

Fig.4. Credit Rating Model Cloud Configuration

\section{Research Conclusion}

This paper draws on the cloud computing technology. From the credit rating of the data collection, model construction, deployment of the system, risk early warning to the credit rating of the application, and the thought of Cloud Architecture are studied. It is found that the cloud computing technology and its application in the field of credit rating are feasible and meaningful to promote the role of innovation and promote the value. 


\section{References}

[1]Li Qiao, Zheng Xiao: Summary of Research on Cloud Computing, Computer Science

[2] Hu Hui, Wang Hui: Analysis on the current situation and development trend of cloud computing technology, Software Guide

[3] Zhao Tingting, Mei Jiandong: Research and solution of security of cloud computing, Computer Security

[4]Zhang Jianwen, Wang Xin: Discussion on the application of cloud computing technology in bank, Southern China financial computer。

[5]Fu Zhihui: Cloud computing implementation problems, Computer knowledge and technology

[6]Zuo Baohe, Luo Xuan: Exploration and Prospect of cloud computing application and practice based on cloud computing theory, Computers and modernization。 